\title{
Experimental and numerical study of spatial and temporal evolution of nonlinear wave groups
}

\author{
L. Shemer and B. Dorfman \\ Tel-Aviv University, Tel-Aviv, Israel
}

Received: 22 August 2008 - Revised: 27 October 2008 - Accepted: 27 October 2008 - Published: 8 December 2008

\begin{abstract}
The evolution along the tank of unidirectional nonlinear wave groups with narrow spectrum is studied both experimentally and numerically. Measurements of the instantaneous surface elevation within the tank are carried out using digital processing of video-recorded sequences of images of the contact line movement at the tank side wall. The accuracy of the video-derived results is verified by measurements performed by conventional resistance-type wave gauges. An experimental procedure is developed that enables processing of large volumes of video images and thus allows capturing the spatial structure of the instantaneous wave field along the whole tank. The experimentally obtained data are compared quantitatively with the solutions of the Modified Nonlinear Schrödinger (MNLS, or Dysthe) equation written in either temporal or spatial form. The adopted approach allows studying evolution along the tank of wave frequency spectra, as well as the temporal variation of the wave number spectra. It is demonstrated that accounting for the 2nd order bound (locked) waves is essential for getting a qualitative and quantitative agreement between the measured and the computed spectra. The relation between the frequency and the wave number spectra is discussed.
\end{abstract}

\section{Introduction}

Rapid advancement in both theoretical and experimental studies of water waves that occurred in recent decades were prompted by the discovery by Benjamin and Feir (1967) of the sideband instability of weakly nonlinear Stokes waves. Important theoretical model for studying the instability and the long time behavior of the nonlinear water waves was developed by Zakharov (1968). The Zakharov integral equation

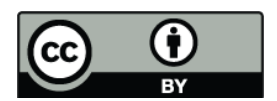

Correspondence to: L. Shemer (shemer@eng.tau.ac.il) describes near-resonant interactions between waves at the lowest possible order and was originally derived for infinitely deep water. Zakharov also showed that under the assumption of narrow wave spectrum, Nonlinear Schrödinger (NLS) equation can be deduced for unidirectional waves from the Zakharov equation. The NLS equation, accurate to the 3rd order in the wave steepness $\varepsilon$, therefore describes resonant interactions pertaining to a weakly nonlinear wave train with a narrow band of frequencies and wave lengths, and governs the slow modulation of the wave group envelope. Later the NLS equation for water gravity waves in water of finite depth was derived by using the multiple scales method by Hasimoto and Ono (1972), and by applying the averaged Lagrangian formulation by Yuen and Lake (1975). In the study of Lake et al. (1977) an agreement was obtained between the experimentally found growth rates of the unstable sidebands and the theoretical predictions based on the NLS equations.

Shemer et al. (1998) performed quantitative comparison of the numerical simulations based on the NLS equation with experiments in a laboratory wave flume. They demonstrated that while the NLS equation is adequate for qualitative description of the global properties of the envelope evolution of unidirectional nonlinear wave groups, such as focusing observed for water waves in sufficiently deep water, it is incapable of capturing more subtle features, for example the emerging front-tail asymmetry observed in experiments. Due to nonlinear interactions, considerable widening of the initially narrow spectrum can occur, violating the spectrum width assumptions of the NLS equation. More advanced models are therefore required for an accurate description of nonlinear wave group evolution. The Modified Nonlinear Schrödinger (MNLS) equation derived by Dysthe (1979) is a higher (4th) order extension of the NLS equation, where the higher order terms account for finite spectrum width, see Stiassnie (1984). Further modification of the NLS equation appropriate for wider wave spectra was presented by Trulsen and Dysthe (1996) and Trulsen et al. (2000). Kit and She-

Published by Copernicus Publications on behalf of the European Geosciences Union and the American Geophysical Union. 
mer (2002) have demonstrated that this modification can be easily derived by expanding the dispersion term in the Zakharov equation into the Taylor series. The effect of each one of the additional (as compared to the NLS equation) terms in the Dysthe model was studied in Shemer et al. (2002). They demonstrated that for steep waves all these terms are essential and contribute significantly to the accuracy of the solution.

The theoretical models mentioned above were derived to describe the evolution of the wave field in time. Complete information on the wave field along the tank at a prescribed instant constitutes the initial condition required for the solution of the problem. In laboratory experiments, however, waves are generated by a wavemaker usually placed at one end of the experimental facility. The experimental data are commonly accumulated using sensors placed at fixed locations within the tank. Hence, to perform quantitative comparison of model predictions with results gained in those experiments, the governing equations have to be modified to a spatial form, to describe the evolution of the temporally varying wave field along the experimental facility. Such a modification of the Dysthe model was carried out by Lo and Mei (1985) who obtained a version of the equation that describes the spatial evolution of the group envelope. Numerical computations based on the Dysthe model for unidirectional wave groups propagating in a long wave tank indeed provided good agreement with experiments and exhibit the front-tail asymmetry, Shemer et al. (2002). The spatial version of the Dysthe equation was also derived by Kit and Shemer (2002) from the spatial form of the Zakharov equation (Shemer et al., 2001, 2007) that is free of any restrictions on the spectrum width.

In the present work, the evolution along the tank of narrow-spectra unidirectional nonlinear wave groups excited by a wavemaker is studied using digital processing of videorecorded sequences of images of the contact line movement at the tank side wall. The technique allows accurate measurements of both the spatial variation of the instantaneous surface elevation along the whole tank, and of the temporal variation of the surface elevation at any prescribed location within the tank. The comparison of the experimentally obtained data thus can be carried out with the solutions of the model equations presented in the temporal or the spatial form. The Dysthe MNLS equation describes evolution of the complex nonlinear wave group envelope and constitutes an appropriate theoretical model for studying such wave groups. The advantages and disadvantages of the spatial and temporal forms of the model equation are discussed. The Dysthe equation in both temporal and spatial forms is solved numerically, and the results of both versions are compared quantitatively with the experimental data.

\section{Theoretical background}

Consider a narrow-banded unidirectional deep-water wave group with the dominant frequency $\omega_{0}$ and wave number $k_{0}$ that are related by the deep-water dispersion relation for gravity waves:

$\omega_{0}^{2}=k_{0} g$

where $g$ is the acceleration due to gravity. Evolution of the wave group in a wave flume can be represented by variation in time and space of either the surface elevation $\eta(x, t)$, or of the velocity potential $\phi$ at the free surface, $\psi(x, t)=\phi(x, z=\eta, t)$. For a narrow-banded wave group it is convenient to express the variation of $\eta$ and $\psi$ at the leading order in terms of their complex envelope amplitudes:

$\eta(x, t)=\operatorname{Re}\left[a_{\eta}(x, t) \exp i\left(k_{0} x-\omega_{0} t\right)\right]$

$\psi(x, t)=\operatorname{Re}\left[a_{\psi}(x, t) \exp i\left(k_{0} x-\omega_{0} t\right)\right]$

The MNLS coupled system of equations, which describes the evolution of the complex envelope $a(x, t)$ and of the potential of the induced mean current $\phi(x, z, t)$ was in fact derived by Dysthe for the surface velocity potential amplitude, $a_{\psi}$ It was demonstrated by Hogan (1986), see also Kit and Shemer (2002), that while at the 3rd order the governing equation for both amplitudes, of the surface elevation, $a_{\eta}$, and of the free surface velocity potential, $a_{\Psi}$, are identical, and thus there is no difference in the NLS equation for either of those amplitudes, at the 4th order the governing equations differ somewhat. For quantitative comparison of the model predictions with the experiment that directly provides data on the surface elevation variation, the equation describing the variation of $a_{\eta}$ is applied in sequel, with the index " $\eta$ " omitted. In fixed coordinates, the governing system of equations has the following form:

$$
\begin{aligned}
& \frac{\partial a}{\partial t}+\frac{\omega_{0}}{2 k_{0}} \frac{\partial a}{\partial x}+i \frac{\omega_{0}}{8 k_{0}^{2}} \frac{\partial^{2} a}{\partial x^{2}}+\frac{i}{2} \omega k^{2}|a|^{2} a-\frac{1}{16} \frac{\omega_{0}}{k_{0}^{3}} \frac{\partial^{3} a}{\partial x^{3}} \\
& +\frac{\omega_{0} k_{0}}{4} a^{2} \frac{\partial a^{*}}{\partial x}+\frac{3}{2} \omega_{0} k_{0}|a|^{2} \frac{\partial a}{\partial x}+i k_{0} a \frac{\partial \phi}{\partial x} z=0=0 \\
& \frac{\partial^{2} \phi}{\partial x^{2}}+\frac{\partial^{2} \phi}{\partial z^{2}}=0 \quad z \quad<=0 .
\end{aligned}
$$

These equations are subject to the boundary conditions at the free surface

$$
\frac{\partial \phi}{\partial z}=\frac{\omega_{0}}{2} \frac{\partial|a|^{2}}{\partial x} \quad(z=0)
$$

and at the bottom

$$
\frac{\partial \phi}{\partial z}=0(z \rightarrow-\infty)
$$

The first four terms in Eq. (3) constitute the cubic Schrödinger equation for deep water in the fixed frame of reference. Dysthe's model is of the 3rd order in the wave 
steepness $\varepsilon$ and can be derived from the 3rd order Zakharov integral equation by adding the narrow-band assumption with spectral width $O(\varepsilon)$, Stiassnie (1984). Incorporation of the narrow-band assumption results in the overall 4 th order Dysthe equation.

The sign of the term $a^{2} \partial a^{*} / \partial x$ in Eq. (3) is positive, while in the velocity potential version used in Dysthe (1979) and Lo and Mei (1985) it is negative. The opposite signs of this term constitute the only difference between the two versions of the 4th order envelope evolution equation.

The problem of wave field evolution in a tank admits two different formulations. In the so-called temporal formulation, the spatial distribution of the complex envelope $a(x)$ is presumed to be known at a prescribed instant $t_{0}$, and its variation in time is obtained by numerical solution of the model equation. Alternatively, the variation of the complex envelope in time, $a(t)$, can be specified at a prescribed location $x=x_{0}$, and the variation of $a(t)$ along the tank is then studied in the spatial formulation using the appropriately modified model equations. It should be stressed that the spatial formulation is routinely applied in the experiment-related studies (Lo and Mei, 1985; Shemer et al., 2002), since the wave gauges provide information on the temporal variation of the surface elevation at fixed locations. The experimental approach of the present study makes it possible to measure the variation with time of the instantaneous complex group envelope along the tank, as well as the variation of the surface elevation with time at any location within the tank. Both temporal and spatial formulations of the Dysthe equation are therefore employed.

Consider first the temporal model. In analogy to Lo and Mei (1985), in a coordinate system moving at the group velocity $c_{g}=\omega_{0} / 2 k_{0}$, the following dimensionless scaled variables are introduced:

$\tau=\varepsilon^{2} \omega_{0} t ; \xi=\varepsilon k_{0}\left(x-c_{g} t\right), A=a / a_{0}, \Phi=\omega_{0} a_{0}^{2} \phi ; Z=\varepsilon k_{0} z$

In these variables, the equations for $A$ and $\Phi$ are:

$$
\begin{aligned}
& \frac{\partial A}{\partial \tau}+\frac{i}{8} \frac{\partial^{2} A}{\partial \xi^{2}}+\frac{i}{2}|A|^{2} A \\
& +\varepsilon\left(-\frac{1}{16} \frac{\partial^{3} A}{\partial \xi^{3}}+\frac{1}{4} A^{2} \frac{\partial A^{*}}{\partial \xi}+\frac{3}{2}|A|^{2} \frac{\partial A}{\partial \xi}+i A \frac{\partial \Phi}{\partial \xi}_{z=0}\right)=0 \\
& \frac{\partial^{2} \Phi}{\partial \xi^{2}}+\frac{\partial^{2} \Phi}{\partial Z^{2}}=0 \quad(Z<0),
\end{aligned}
$$

with $\Phi$ satisfying the following boundary conditions:

$$
\frac{\partial \Phi}{\partial Z}=\frac{1}{2} \frac{\partial|A|^{2}}{\partial \xi}, \quad Z=0, \frac{\partial \Phi}{\partial Z}=0, Z \rightarrow-\infty
$$

Equations (7) to (10) and the appropriate initial conditions constitute the temporal version of the Dysthe model. The corresponding spatial version can be obtained either from Eq. (3) as in Lo and Mei (1985), or from the spatial version of the Zakharov equation, Kit and Shemer (2002). The scaled dimensionless space and time variables in Eq. (7) are replaced for the spatial version by

$$
\xi=\varepsilon^{2} k_{0} x, \tau=\varepsilon \omega_{0}\left(x / c_{g}-t\right)
$$

The governing equations then assume the following form

$$
\begin{aligned}
& \frac{\partial A}{\partial \xi}+i \frac{\partial^{2} A}{\partial \tau^{2}}+i|A|^{2} A+8 \varepsilon|A|^{2} \frac{\partial A}{\partial \tau}+2 \varepsilon A^{2} \frac{\partial A^{*}}{\partial \tau} \\
& +\left.4 i \varepsilon A \frac{\partial \Phi}{\partial \tau}\right|_{Z=0}=0 \\
& 4 \frac{\partial^{2} \Phi}{\partial \tau^{2}}+\frac{\partial^{2} \Phi}{\partial Z^{2}}=0 \quad Z<0 \\
& \left.\frac{\partial \Phi}{\partial Z}\right|_{Z=0}=\frac{\partial|A|^{2}}{\partial \tau}, \quad \frac{\partial \Phi}{\partial Z}=0, \quad Z \rightarrow-\infty
\end{aligned}
$$

The formulation of the spatial model given by Eqs. (11)-(14) is completed by specifying the temporal variation of the envelope at the prescribed location $A\left(\xi_{0}, \tau\right)$.

In both the temporal and the spatial formulations, the normalized envelope shape $A(\xi, \tau)$ determines the surface elevation at the leading order. With $A(\xi, \tau)$ known, application of Eq. (2a) represents the so-called free waves only. The 2nd order higher frequency bound, or locked, waves can also be determined from $A(\xi, \tau)$ using:

$B(A)=\frac{1}{2} \varepsilon A^{2}$

The surface elevation that contains the 2 nd order bound waves with frequencies and wave numbers that are respectively twice higher than those of the free waves are thus obtained for both temporal and spatial formulation as

$\eta / a_{0}=\operatorname{Re}\left(A e^{i\left(k_{0} x-\omega_{0} t\right)}+B(A) e^{2 i\left(k_{0} x-\omega_{0} t\right)}\right)$

\section{Experimental facility and the initial conditions}

The experiments were performed in the wave tank that is $18 \mathrm{~m}$ long, $1.2 \mathrm{~m}$ wide and has transparent side walls, as well as windows at the bottom which allow viewing of the flow from various directions. The tank is filled to mean water depth of $0.6 \mathrm{~m}$. Waves are generated by a computercontrolled paddle-type 4-module wavemaker that is placed in sealed boxes within the tank, so that the paddles are located at the distance of about $1 \mathrm{~m}$ from the tank end. Beach for wave energy absorption starts at the distance of about $3 \mathrm{~m}$ from the other end of the tank. The net length of the facility therefore is about $14 \mathrm{~m}$. The instantaneous surface elevation at any fixed location can be measured by resistance type wave gauges made of $0.3 \mathrm{~mm}$ platinum wire. The gauges are shifted along the tank using an instrument carriage. More details about the facility can be found in Shemer et al. (2007).

The wave gauges in this study were applied mainly for validation of the accuracy of surface elevation measurements 
by digital processing of video clips that record the contact line movement at the tank's wall. The instantaneous contact line shapes were recorded by a single monochrome CCD video camera (Pixelink PL-A471) at a rate of $30 \mathrm{fps}$. The size of each frame is 640 by 480 pixels. The field of view of the camera located one meter from the tank wall spans $50 \mathrm{~cm}$ along the tank, yielding the pixel dimension of about $0.8 \mathrm{~mm}$. Advantage was taken of extremely high repeatability of the wave field emanating from the prescribed wavemaker driving signal. The camera is placed on the instrument carriage to enable imaging of different regions of the tank. Each camera recording session is synchronized with the wavemaker driving signal using a common reference signal. A single wave group was generated for each recording session. For the consecutive recording session, the carriage is shifted along the tank, so that slightly overlapping images of the contact line movement along the whole experimental facility are obtained. Every frame of the recorded video clip at each camera location was processed separately.

Experiments were performed for a wave group with Gaussian envelope generated by the wave maker. The temporal variation of the surface elevation at the wavemaker has to the leading order the following form:

$\eta(t, x=0)=a_{0} A(t) \cos \left(\omega_{0} t\right)$

with the Gaussian envelope shape given by

$A(t, x=0)=e^{-\left(\frac{t}{m T_{0}}\right)^{2}}$.

The selection of the wave parameters in Eqs. (17) and (18) was based on the following considerations. Shorter dominant wave length $\lambda_{0}$ increases the effective length of the facility (in terms of $\lambda_{0}$ ) and thus allows studying group evolution over extended range. On the other hand, if the dominant wave is too short, the shape of wave groups prescribed by Eqs. (17) and (18) can not be faithfully reproduced in the present facility. Shorter waves also have lower absolute amplitudes, resulting in larger relative measurement errors. The dominant wave period selected in the present experiments $T_{0}=0.7 \mathrm{~s}$ corresponds to the wave length $\lambda_{0}=2 \pi / k_{0}=0.76 \mathrm{~m}$. The initial width of the group is determined by the parameter $m$. The group becomes wider as the value of $m$ increases; correspondingly, the surface elevation frequency spectrum becomes narrower with $m$ increased. Based on our earlier studies (Shemer et al., 2002), the value of $m=3.5$ was chosen. This value of $m$ corresponds to the spectral width that is sufficient to satisfy the narrow spectrum constraint for the applicability of the Dysthe equation. On the other hand, the extension of the group for $m=3.5$ is short enough to enable studying of the temporal evolution of the group within the tank.

For a given dominant wave period, the value of the wave amplitude $a_{0}$ in Eq. (17) determines the maximum steepness of the wave group $\varepsilon=a_{0} k_{0}$. To enable determination of the instantaneous spatial envelope shape of the wave group and to study its nonlinear temporal evolution, the entire group has to be present in the tank. Hence, on one hand, the group generation by the wavemaker has to be completed before initiation of the study of the temporal variation of the envelope shape, and on the other hand, measurements of spatial wave group structure remain meaningful as long as the front of the group does not reach the beach. The group propagates along the tank with the group velocity $c_{g}=0.54 \mathrm{~m} / \mathrm{s}$. The spatial extension of the group for the adopted parameters does not exceed 6-7 $\mathrm{m}$. When the generation of the group by the wavemaker is completed, the group front is about $5 \mathrm{~m}$ from the beach, leaving the duration that does not exceed $10 \mathrm{~s}$ to study the wave group evolution before its front reaches the far end of the tank. According to Eq. (7), the time scale of the nonlinear effects is $O\left(\varepsilon^{2}\right)$. Hence, for the duration of the process prescribed by the group shape, the dominant frequency and the length of the tank, higher wave steepness increases the effective evolution time at the slow scale $\tau$. On the other hand, nonlinear waves with higher steepness tend to break. Since wave breaking cannot be accounted for by the Dysthe model, the wave steepness must remain below the value that can lead to wave breaking.

The maximum initial wave steepness of $\varepsilon=0.18$ $\left(a_{0}=22 \mathrm{~mm}\right)$ adopted in this study was selected experimentally on the basis of visual observations of wave group propagation along the tank with different values of $a_{0}$. For this value of $\varepsilon, \tau=1$ corresponds to dimensional duration $t=3.44 \mathrm{~s}$, or 4.9 dominant wave periods. This is well below the experiment duration limit of about $7 \mathrm{~s}$ imposed by the effective length of the tank. The spectral width of the signal given by Eq. (18), calculated as in Shemer et al. (2002), is $\Delta \omega / \omega_{0}=0.054<\varepsilon$, thus satisfying the narrow spectrum limit of the Dysthe model. For these experimental conditions, the dimensionless depth $k_{0} h \approx 5$, so that the condition for the validity of the Dysthe model, $k_{0} h=O(1 / \varepsilon)$ is also satisfied.

The experimental results are compared with the theoretical predictions based on the numerical solution of the Dysthe equation in either temporal, Eqs. (7)-(10), or spatial, Eqs. (11)-(14), forms, together with the corresponding initial conditions. The initial envelope shape $A(0, \tau)$ for the spatial evolution case is given by Eq. (18) with the scaling defined by Eq. (11).

In the spatial evolution formulation the initial condition emerges naturally from the water surface elevation variation in time excited by a wavemaker located at $x=0$. Contrary to that, in the temporal evolution case the initial conditions defining the wave field in the whole tank are to be prescribed at a certain instant. One possibility to define the initial conditions for the temporal formulation of the problem is to use the actually measured instantaneous wave field when the whole group emerges in the tank. For a relatively short wave tank used in the present study this option, however, restricts severely the duration of the wave group evolution and thus the role of nonlinearity which constitute the main interest of the present study. 
An alternative approach was therefore employed. Since nonlinear effects become prominent at slow scales, cf. Eqs. (7), (11), it can be assumed that the initial evolution of the wave group is mainly governed by linear dispersion effects, while nonlinearity can be neglected. This assumption enables linearization of the governing Eq. (12), yielding

$$
\frac{\partial A}{\partial \xi}+i \frac{\partial^{2} A}{\partial \tau^{2}}=0
$$

Following Pelinovski and Kharif (2000), the solution of Eq. (19) for a Gaussian envelope at the wavemaker given by Eq. (18) can be written in the physical variables $(x, t)$ as

$A(x, t)=|A(x, t)| \exp (i \theta)$

where the amplitude $|A(x, t)|$ and the phase $\theta$ of the envelope are given by

$$
\begin{aligned}
& |A(x, t)|=\frac{m \pi}{\sqrt[4]{m^{4} \pi^{4}+4 k_{0}^{2} x^{2}}} \exp \\
& -\left[\frac{m^{2} \pi^{2}}{4\left(m^{4} \pi^{4}+4 k_{0}^{2} x^{2}\right)}\left(\omega_{0} t-2 k_{0} x\right)^{2}\right],
\end{aligned}
$$

$\theta=\frac{k_{0} x\left(\omega_{0} t-2 k_{0} x\right)^{2}}{2\left(m^{4} \pi^{4}+4 k_{0}^{2} x^{2}\right)}-1 / 2 \tan ^{-1}\left(\frac{2 k_{0} x}{m^{2} \pi^{2}}\right)$.

While Eqs. (20)-(21) represent the solution of the spatial evolution problem; they describe the complex group envelope variation in time and space, and thus can be used to define the initial conditions for the solution of the temporal evolution problem. The calculated according to Eq. (21) envelope shape is presented in Fig. 1a at two instants. The 1st curve corresponds to the instant when the maximum of the envelope is at the wavemaker located at $x=0$. The 2 nd curve represents the spatial distribution of the group envelope immediately before the entrance of the group to the tank and corresponds to the instant when the wave group excitation by the wavemaker is initiated in the experiments. This wave envelope is somewhat wider than the 1 st one, with the maximum value below unity. This complex envelope shape prior to the group's "entrance" to the tank served as the initial condition. Time in the present study is thus measured relative to that instant of initiation of the wavemaker movement. The wavenumber spectrum of the surface elevation presented in Fig. 1b apparently does not vary in time for the linearized problem and therefore can be seen as the initial spectrum for the nonlinear evolution problem.

The Dysthe model describing either the spatial or the temporal evolution of a nonlinear wave group is solved using the pseudo-spectral split-step Fourier method based on Lo and Mei (1985). The computed complex envelope is then translated into the physical coordinates $(x, t)$. The variation of
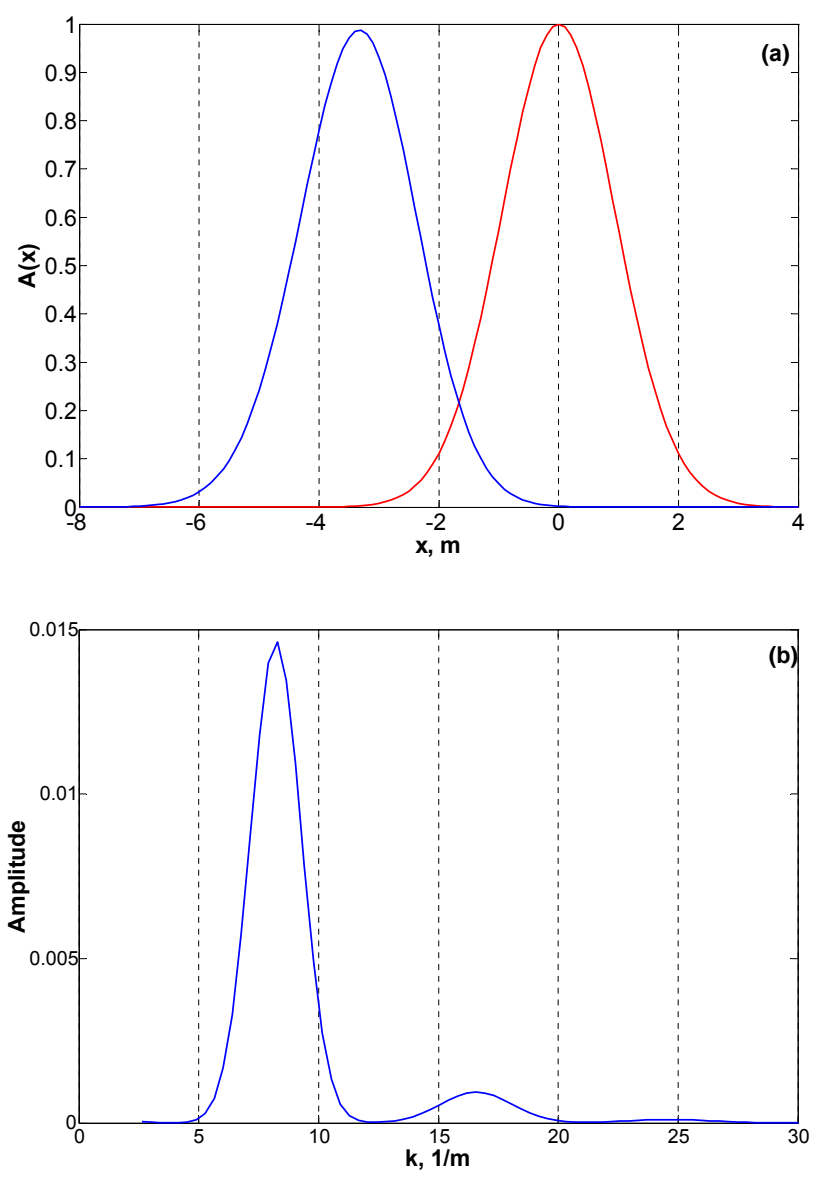

Fig. 1. Initial conditions for the temporal evolution computations. (a) Envelope shape centered at the wavemaker $(x=0 \mathrm{~m})$ and at the instant corresponding to initiation of the wavemaker operation; (b) Initial wavenumber amplitude spectrum.

the surface elevation at any fixed location $\eta\left(x_{0}, t\right)$ in the spatial formulation, or at the fixed instant $\eta\left(x, t_{0}\right)$ in the temporal formulation, is obtained from the complex envelope that contains the $2^{\text {nd }}$ order correction calculated using Eq. (16).

\section{Video data processing}

An example of a recorded image is presented in Fig. 2. While the contact line can be clearly identified visually, the image contains numerous additional features such as the tank supporting beam, objects in the laboratory beyond the tank, reflections, etc. An effective algorithm was developed to extract quantitative information from the recorded video clips that contain thousands of images like that in Fig.2.

The images were first preprocessed using contrast enhancement and linear filtering (see, e.g. Gonzales et al., 2004). Search of the contact line coordinates is carried out in the vicinity of the contact line and is thus unaffected by other spurious curves that may appear in the image. A searching 


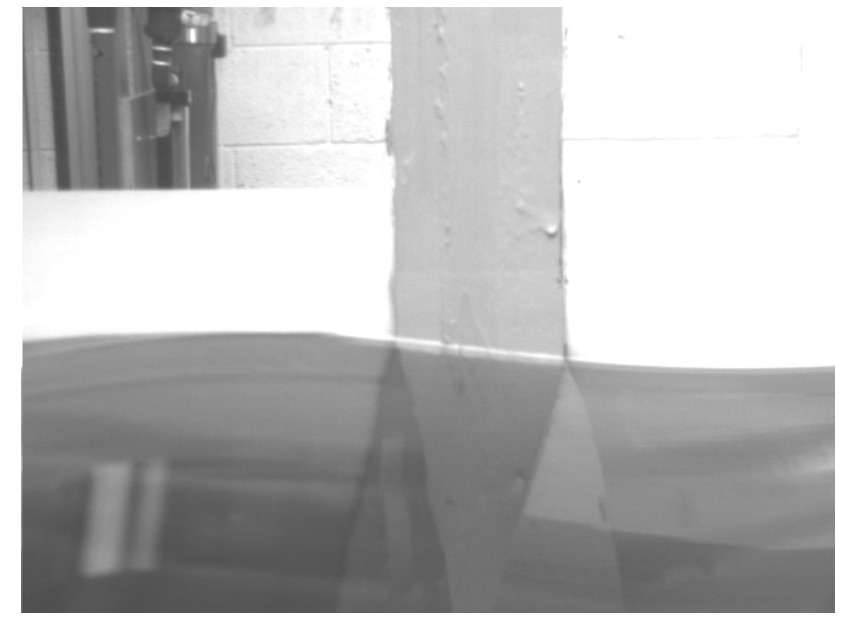

Fig. 2. Contact line image that contains tank wall supporting beam and the viewing window.

area is cropped from the whole image in the vicinity of the desired curve as a rectangular window that is small with respect to the entire image. The initial window is built in the 1st image of the series around a point that constitutes the center of the searching area and is chosen at or in a close vicinity to the desired curve. Since the slope of the interface is usually quite small, the window aspect ratio selected in most cases is in the range of 2 to 3 , with the width of the window being about 50 pixels.

The vertical coordinate of the interfacial curve for every horizontal location is defined as the weighted average of the pixel intensities along the vertical extent of the window. Once all vertical coordinates within the window are calculated, contact line shape within the window is approximated by a second order polynomial using the least mean square fit on the array of the detected points. The vertical coordinate of the contact line at the center of the window is finally obtained from the polynomial value at the corresponding horizontal coordinate. The contact line coordinates determined by this procedure contain contribution of the pixel intensities in the vicinity of each point and are obtained at a sub-pixel resolution.

The window is then shifted forward by one pixel in the direction given by the slope of the contact line, and the process is repeated. At each step, the window is inclined by an angle corresponding to the window shift direction. This process continues until the whole image is covered. Once the coordinates of the contact line profile in a given frame have been determined, the search in the next frame is performed utilizing this information. At the recording rate of 30 frames per second, the contact line shift between the consecutive frames is quite small, making it advantageous to start the search in the next frame with the initial window built around the previous profile.
The applied procedure has an additional advantage of allowing processing of numerous clips captured during the experiment at different locations along the tank automatically. At various locations along the tank the vertical position of the camera and its distance from the opposite tank wall remain constant within a reasonable accuracy. Each clip was recorded after the camera has been shifted along the tank by the distance corresponding to the horizontal extent of the recorded image, and the wavemaker was activated after a sufficient delay so that all waves from the previous run have vanished. The initial search window in the consecutive clips is placed according to the coordinate of the interface determined in the last window of the clip recorded at the upstream location at the identical timing relative to the reference signal.

The present experimental approach was validated extensively using conventional resistance wire gauges at a number of locations along the tank and comparing with data simultaneously acquired at same distance $x$ by image processing technique. Measurements of the evolution of wave groups with wide frequency spectra that vary significantly along the tank due to dispersion and nonlinearity (Shemer et al., 2007) were performed for validation purposes. The spanwise uniformity of the surface elevation was also checked by placing the probes across the tank. The difference between the instantaneous values of the surface elevation measured by the wave gauge located close to the tank's wall and by video image processing at various distances from the wavemaker always remains well below $1 \mathrm{~mm}$ and does not exceed the deviation between the outputs of different probes. The spectra derived from those measurements exhibit a very good agreement for all frequencies in the spectra. More details on the experimental method employed are given in Dorfman and Shemer (2007).

\section{Experimental and numerical results}

The temporal variation of the surface elevation within a wave group with the initially Gaussian envelope at $x=0$ as given by Eqs. (17) and (18) is studied first. Variation of the surface elevation within the group at a number of locations along the tank computed according to Eqs. (11)-(14) is compared in Fig. 3 with the results of video image processing.

The computed values of $\eta(t)$ in Fig. 3 contain the contribution of the bound waves, see Eqs. (15) and (16). The shift in the horizontal scale in the consecutive frames of Fig. 3 reflects the time elapsed while the group traveled between the measuring stations. Excellent agreement is obtained between the experimental results and the computations based on the spatial evolution version of the Dysthe model, Eqs. (11) (14). No measurements were performed in the immediate vicinity of the wavemaker due to contamination of the wave field in this region by evanescent standing waves, see Dean and Dalrymple (1991). The sequence of frames in Fig. 3 

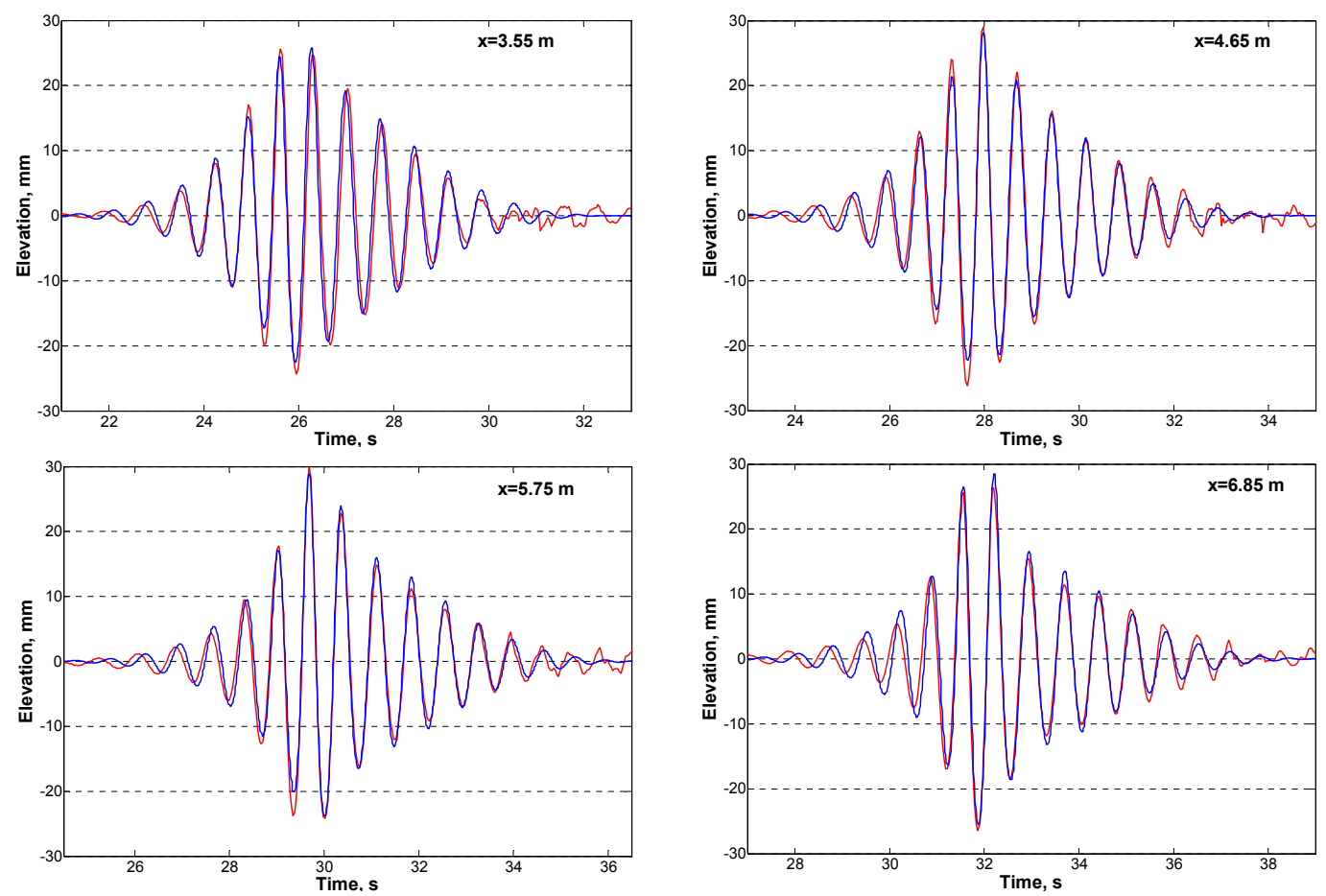

Fig. 3. Variation of the surface elevation within the group at different distances $x$ from the wavemaker: - experiments; - simulations.

clearly demonstrates that the duration of the group extends with $x$ and that the initially symmetric Gaussian envelope shape (Fig. 1a) gradually exhibits stronger left-right asymmetry, with increasingly steep front and elongated tail. The maximum surface elevation within the group may exceed significantly the nominal value of $a_{0}$. This increase of the maximum amplitude is associated in part with the focusing properties of the nonlinear Schrödinger equation that affects the shape of the group envelope, as discussed in Shemer et al. (1998). The NLS equation, however, is only capable of reflecting correctly some limited features of the solution, and the extension to the MNLS equation is required to get both qualitative and quantitative agreement between experiments and computations, see Shemer et al. (2002). Ap apparent additional reason for higher maximum values of the surface elevation notable in Fig. 3, as well as for the crest-trough asymmetry, is the contribution of 2 nd order bound (locked) waves.

The notable variation of the group shape along the tank in Fig. 3 is due to both linear dispersion and nonlinear effects. To separate linear and nonlinear contributions, frequency spectra of surface elevation variation in time that vary only due to nonlinear effects, are presented in Fig. 4. The frequency spectra of Fig. 4 are plotted for the same locations along the tank as in Fig. 3. The spectra are computed for those parts of the surface elevation records that contain the whole group with duration of about $13 \mathrm{~s}$ (about 20T $T_{0}$ ). The spectra are thus discrete with the frequency increment of about $0.077 \mathrm{~Hz}$. For demonstration purposes only, the amplitude spectra obtained for the computed temporal variation of the surface elevation that naturally are smoother than the results derived from the experimental data, are drawn as a solid line.

The agreement between experiments and computations in Fig. 4 is quite good. While the initial amplitude spectrum corresponding to Eq. (17) also has a symmetric Gaussian shape, the spectra of Fig. 4 are asymmetric and deviate from the Gaussian shape. Note the existence of a kink in the spectral shape at the frequency slightly exceeding the dominant one, $f_{0}=1 / T_{0}=1.43 \mathrm{~Hz}$, that is visible at $x=5.75 \mathrm{~m}$ and becomes stronger at $x=6.85 \mathrm{~m}$. The kink is observable both in the measured and in the computed spectra. Even for a relatively short extent of the spatial evolution, widening of the spectrum is visible in Fig. 4. This spectral widening and nonGaussian spectral shape indicate that nonlinearity is essential in the wave group evolution along the tank.

The contribution of the 2nd order bound waves to the amplitude spectrum is quite significant at all locations. The measured using the digital processing of the video images spectrum of bound waves around the 2nd harmonic of the dominant frequency $f_{0}$ is in excellent agreement with the model predictions. The bound waves' spectrum also becomes wider with the distance from the wavemaker, in agreement with the variation of the free wave spectrum around the dominant frequency $f_{0}$. 

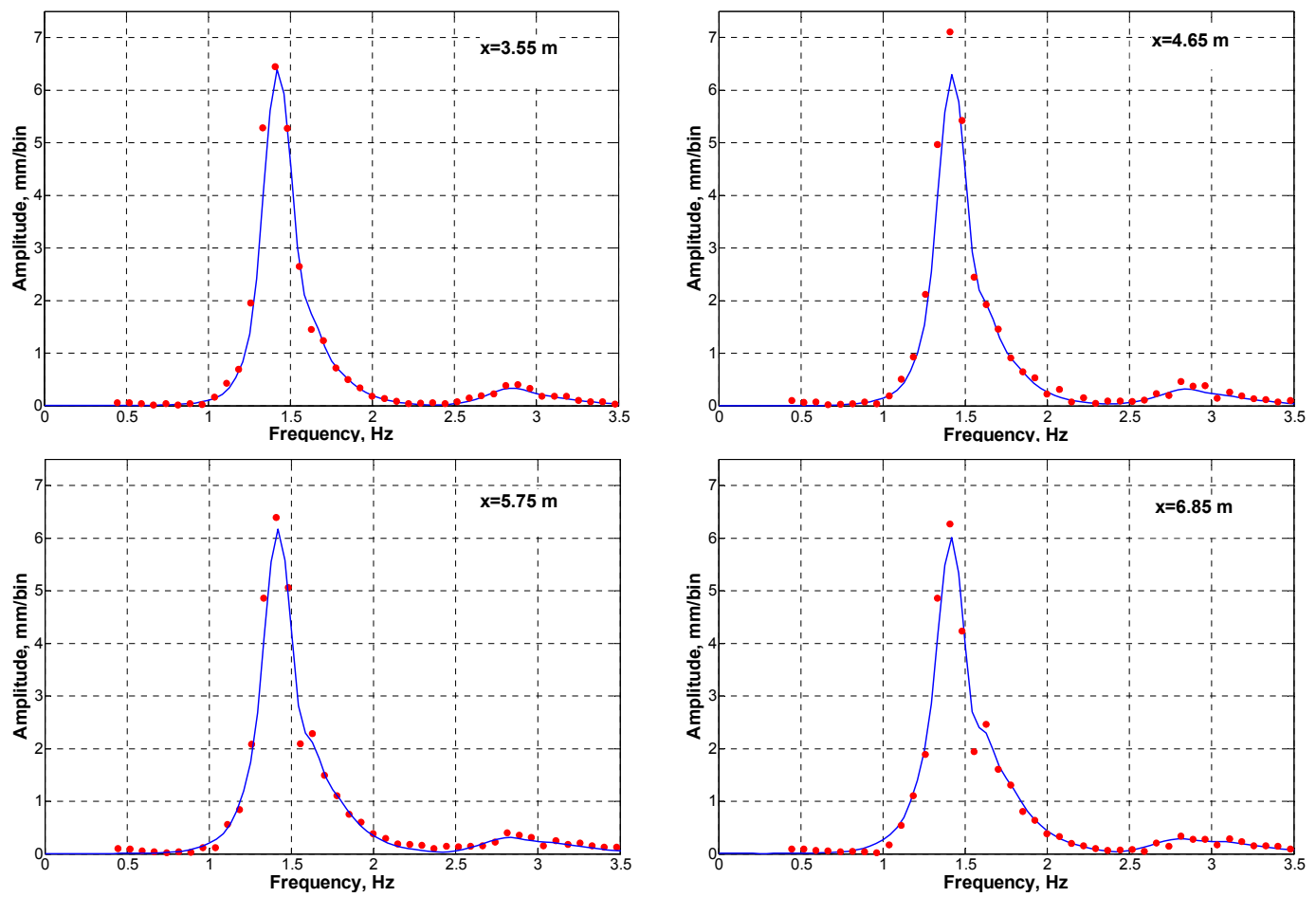

Fig. 4. Variation of the frequency spectra along the tank: symbols - experiments, line - simulations.

As stressed above, the main motivation for developing the data acquisition method based on the processing of sequences of video images is in its capability to measure instantaneous spatial distribution of the surface elevation. Application of this method enables following the temporal evolution of the whole wave group as well. This information can be compared with the numerical solution of the system of Eqs. (7)-(10) that constitute the Dysthe model in its temporal formulation. The initial conditions for the temporal evolution case $A(x, 0)$ are obtained using Eqs. (21) and (22), as described in Sect. 3 and presented in Fig. 1.

To compare the numerical and the experimental results, the whole group at the selected instants has to be physically present within the wave tank boundaries. The numerical solution of Eqs. (7)-(10) indicates that at the dimensional time $t=12 \mathrm{~s}$ (relative to the instant depicted in Fig. 1) the advancement of the group along the tank is sufficient for the tail of the computed instantaneous spatial envelope distribution to emerge within the tank, thus enabling comparison with the experiment. Similarity of the numerical and the experimental results is examined also at three additional instants: $t_{2}=14 \mathrm{~s}$; $t_{3}=16 \mathrm{~s}$ and $t_{4}=18 \mathrm{~s}$. Equations (15)-(16) are used again to account for the contribution of the 2 nd order bound waves.

The spatial variation of the surface elevation as a result of the temporal evolution of the complex wave envelope is presented at the selected instances in Fig. 5. As in the spatial evolution case, good agreement is obtained between the numerical simulations and the experimental observations. At the earliest instant presented in Fig. 5, $t=12 \mathrm{~s}$, the formation of the group has just been completed and the group in its entirety emerges in the tank, while at the last instant, $t=18 \mathrm{~s}$, the front of the group approaches the far end of the wave tank.

Deviation of the group shape in Fig. 5 from the initial envelope presented in Fig. 1a is obvious. Both left-right and trough-crest asymmetries observed in the temporal records presented in Fig. 3, as well as significant variations in the extreme values of the surface elevation within the group, are visible in Fig. 5 as well. Note, however, that the left-right asymmetry in Fig. 5 is opposite to that of Fig. 3, where the steeper part of the group appears at earlier sampling times. The experimental results are in agreement with the numerical solutions of the temporal Dysthe model.

Comparison of Figs. 3 and 5 also illustrates the well known fact that since the group velocity of deep water waves is a half of their phase velocity, the number of waves within the group in the temporal surface elevation variation records of Fig. 3 is twice larger than in the instantaneous spatial "snapshots" of the same group plotted in Fig. 5.

The wave-number amplitude spectra corresponding to Fig. 5 are presented in Fig. 6. The spectra based both on the experimental data and on the numerical simulations were computed for instantaneous surface elevation distributions that cover $12 \mathrm{~m}$ of the tank and contain the whole group. This longitudinal extent of the "snapshot" determines the wave number resolution of the resulting discrete spectra. As in Fig. 4, the spectra derived from the numerical solutions of the MNLS model are plotted as solid lines. 

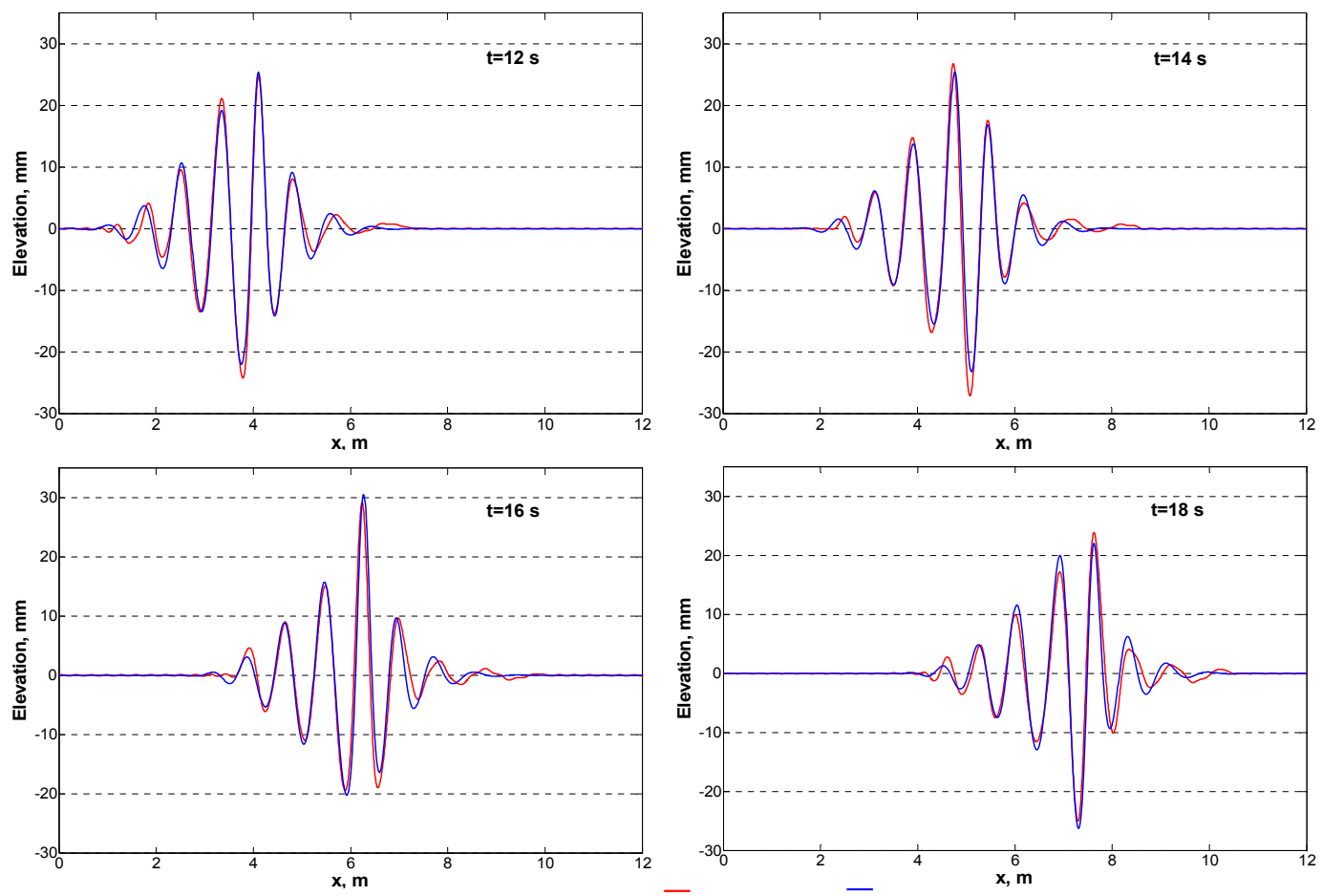

Fig. 5. The instantaneous surface elevation at various instants: — experiments; - simulations.
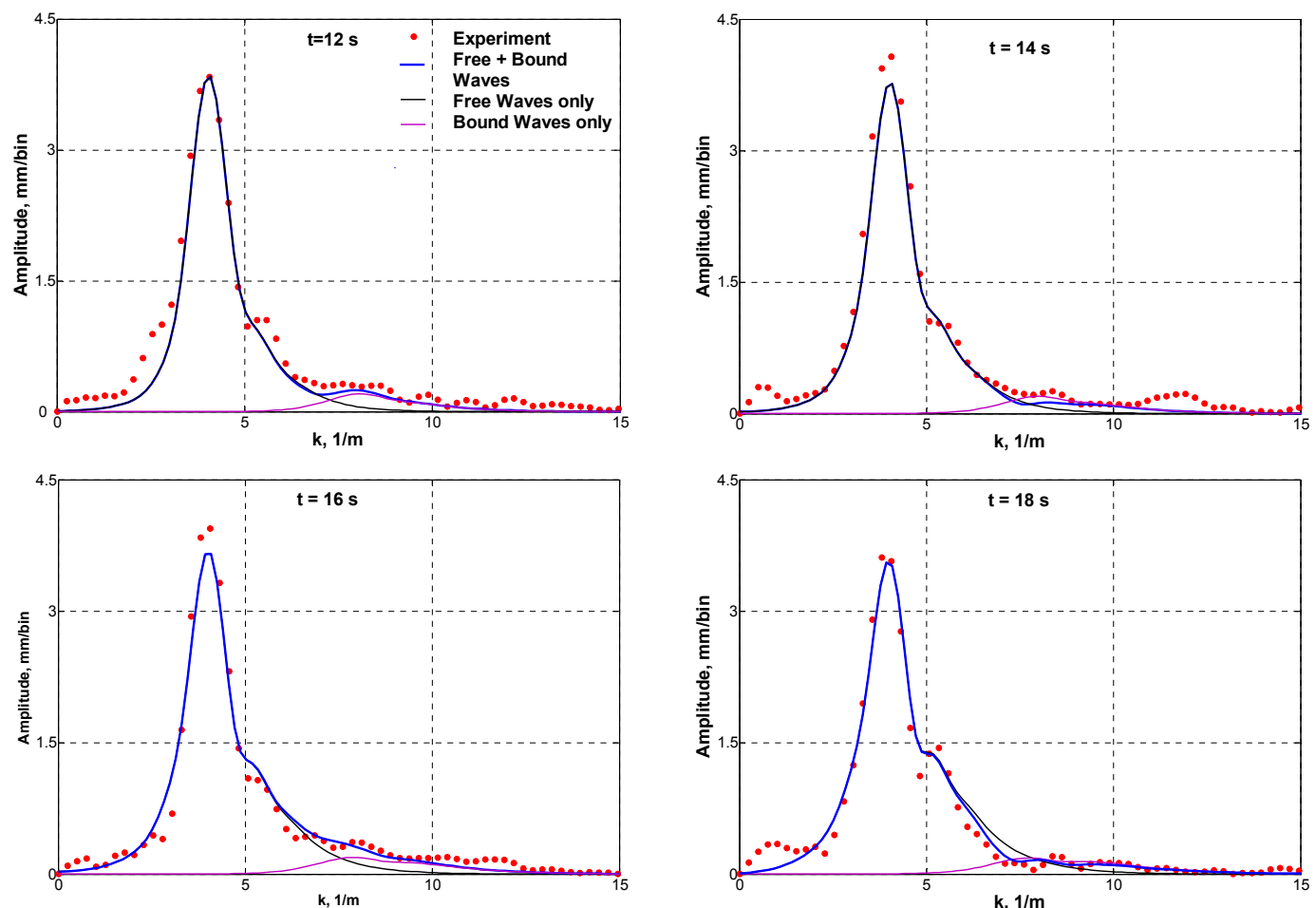

Fig. 6. Wavenumber spectra of the surface elevation at various instants: symbols - experiments; lines - simulation. 
All spectra in Fig. 6 exhibit essential differences from the initial wavenumber spectrum presented in Fig. 1b. The agreement between the simulated and the experimental results in Fig. 6 is quite good at all instances presented; the differences can be attributed in part to inaccuracy associated with choosing the initial condition. There are similarities but also essential differences between the frequency spectra given in Fig. 4, and the wave number spectra of Fig. 6. In both figures the spectra become wider in the course of the wave group evolution. The wave number spectra in all frames of Fig. 6 are however much wider than the frequency spectra in Fig. 4.

The larger width of the wave number spectra relative to the frequency spectra follows from the dispersion relation for deep water, Eq. (1) that is appropriate for the present experiments. In the narrow spectrum approximation the relative widths of those spectra are related by

$$
\frac{\Delta k}{k_{0}}=2 \frac{\Delta \omega}{\omega_{0}}
$$

As a result, in all frequency spectra of the temporal variation of the surface elevation for a narrow-band wave group moving along the tank, Fig. 4, the free waves and the bound waves are totally separated. For the initial condition presented in Fig. 1b, the separation of free and bound waves' spectral domains still exists. The spectral widening in course of group propagation along the tank, however, leads to overlapping domains of the free and of the 2nd harmonic bound waves in the wave number spectra of Fig. 6. Each measured spectrum apparently contains free as well as bound waves. In numerical simulations, complex group envelope that corresponds to free waves only is computed first. Bound waves are then obtained from the free wave field. The computed wave number spectra of free and bound waves are also plotted in Fig. 6 .

The overlapping of free and bound waves domains in the wave number spectra precludes straightforward filtering out of the free wave spectrum from the experimental results. This difficulty complicates significantly the determination of the spatial group envelope's shape that contains the free-wave part only from the experimental data. The initial conditions for solving the temporal evolution problem could not therefore be determined from experiment. This difficulty forced to apply the linearized approach presented by Eqs. (19)-(22) in order to translate the temporal variation of the surface elevation at the wavemaker given by Eqs. (17), (18) into the spatial form.

Accounting for the 2nd harmonic bound waves is essential to get a better agreement with the measured spectra at high wave numbers. The disagreements between computations and measurements in the low wave number region of the spectrum in Fig. 6 may partially stem from the fact that for longer waves, the depth of the experimental facility of $0.6 \mathrm{~m}$ is not large enough for those wave components to be considered deep. The low wave number bound waves may become significant and can constitute a significant contribu- tion to the spectral shape. The effect of long bound waves was considered in the framework of the Zakharov equation in Shemer et al. (2007). The validity of Dysthe equation that served as the theoretical model in the present study, however, is restricted to deep waves. The long bound waves were therefore not considered in the current study.

\section{Discussion}

It should be stressed that theoretical studies of nonlinear water-waves are often performed by solving temporal evolution models, while in the laboratory as well as in field experiments surface elevation variation with time is usually recorded at fixed locations, sometimes these data also contain information on the wave propagation directions. Attempts are sometimes made to translate the measured by point sensors frequency spectrum into the corresponding wave number (or wave vector in the two-dimensional case) spectrum. However, direct quantitative comparison of the frequency and the wave number spectra can not be carried out in a consistent way.

Instantaneous "snapshot" of the whole wave field taken to get the wave number spectrum, on one hand, and measurements of the temporal variation of the surface elevation variation with time at a fixed location to get the wave frequency spectrum, on the other hand, constitute essentially different ways of studying an evolving in space and in time waterwave field. Moreover, as the present study demonstrates, direct computation of the wave number spectrum from the measured frequency spectrum can not be carried out even assuming that the evolution is slow as compared to the relevant temporal scale (represented by the duration of continuous sampling that determines the frequency resolution of the spectrum) and the spatial scale (the extent of the imaged wave field that determines the wave number spatial resolution) of the data acquisition process.

The linear dispersion relation between wave frequencies and wave numbers only holds for the free wave components. Therefore, it is possible in principle to relate quantitatively frequency and wave number spectra for the free wave domain only. Comparison of the frequencies and wave numbers that correspond to the spectrum peaks and clearly represent free waves in Figs. 4 and 6, respectively, indeed reveals that they are related by Eq. (1). The present results, however, demonstrate that in general separation of the wave number spectra into free and bound waves is not always possible. For a relatively narrow initial free-wave spectrum, the high frequency/wave number part of the spectrum consists almost exclusively of bound components. The calculation of spectral bound components from the free wave spectrum is often not straightforward and requires information on the phases of each free wave component. Such information is usually not readily available in reported data on the experimentally measured frequency spectra. It thus appears virtually impossible 
to evaluate quantitatively the shape of the high end of the wave number spectrum from the measured surface elevation variation in time at a fixed location and the corresponding frequency spectrum.

Two version of the MNLS equation are considered here: The first is based on the original formulation of Dysthe (1979), Eqs. (7) to (10), that describes evolution in time of a unidirectional narrow-spectrum wave group with prescribed initial spatial distribution of the complex group envelope. Lo and Mei (1985) were the first to notice that in order to carry out comparison with experimental data provided by point sensors, a version of the MNLS equation that describes evolution of the wave group envelope in space is required. The spatial version introduced in their paper requires prescribed temporal variation of the complex wave envelope at a given location, usually at the wavemaker, as the initial condition.

Each version of the MNLS equation, either spatial or temporal, yields variation of the wave field in time as well as in space. The derivation of the spatial MNLS version by Lo and Mei was based on the temporal Dysthe equation and the appropriate change of variables. The two version of the MNLS equation can also be derived from the corresponding temporal (Stiassnie, 1984; Hogan, 1986) or spatial (Kit and Shemer, 2002) versions of the Zakharov equation. These derivations shed light on two important facts. First, the evolution equations for complex envelopes of the surface elevation variation and for the velocity potential are somewhat different at the 4th order appropriate for the MNLS model. Since the surface elevation is the parameter measured directly, the surface elevation version of the MNLS equation is used here for carrying out quantitative comparison of model predictions with the experimental results.

The second comment is related to the inclusion of additional linear terms in the temporal version of the MNLS equation by Trulsen and Dysthe (1996) and Trulsen et al. (2000). Derivation of the Dysthe model from the Zakharov equation clearly demonstrates that these additional terms appear due to expansion of the interaction coefficient in the Zakharov equation around the carrier wave frequency $\omega_{0}$ in terms of the wave number deviation from $k_{0}$. For unidirectional waves in deep water this expansion has an infinite number of terms and therefore has to be truncated. In the spatial evolution case the situation is different and the expansion is of the wave numbers around the frequency $\omega_{0}$. For the dispersion relation presented by Eq. (1) the expansion does not contain terms beyond quadratic. In the spatial version of the unidirectional MNLS the dispersion is thus presented exactly. The spatial evolution version of the MNLS equation is therefore more accurate in this sense than the temporal version.

\section{Conclusions}

An effective method for identification of the instantaneous contact line shapes in a sequence of recorded video images is developed. Experiments indicate that the surface elevation values calculated employing the proposed image-based method have an error comparable to that of conventional resistance type gauges.

Advantage was taken of the controlled and repeatable character of the experiments that enabled synchronization of the video camera and the wavemaker operation to obtain phase-locked surface elevation distributions on length scales exceeding the size of the imaged scene. The applied technique allows studying temporal evolution of the instantaneous wave field in the whole tank and thus determination of the variation in time of the wavenumber spectra. This ability is important for carrying out quantitative comparison of prediction of nonlinear evolution models with the experimental results.

This experimental approach is adopted here to study both the spatial and the temporal evolution of narrow-banded unidirectional wave groups. The experimental results are compared in detail with the solutions of the appropriate version of the MNLS equation. The present experimental and numerical study demonstrates that the envelopes of deep-water unidirectional wave groups with narrow spectrum have certain similarities in their evolution pattern in both time and space. Good quantitative and qualitative agreement between measurements and computations is obtained for both the spatial and the temporal evolution formulations. The most visible feature of the evolution process is the gradual transformation of the initially symmetric envelope shape into a strongly asymmetric one. This feature can not be reproduced by the cubic Schrödinger equation in which the initially symmetric envelope can not become asymmetric, and requires the extension to the MNLS equation for its proper description.

Both the spatial and the temporal version of the model describe correctly the widening of the spectrum in the course of evolution. The shapes of the spectra are, however, quite different in these formulations, the wave number spectrum being twice wider than the corresponding frequency spectrum.

Acknowledgement. The support of this study by a grant \# 964/05 from the Israel Science Foundation is gratefully acknowledged.

Edited by: V. Shrira

Reviewed by: two anonymous referees

\section{References}

Benjamin, T. B. and Feir, J. E.: The disintegration of wavetrains on deep water, J. Fluid Mech., 27, 417-430, 1967.

Gonzales, R. C., Woods, R. E., and Eddins, S. L.: Digital Image Processing Using MATLAB, Upper Saddle River, NJ: Pearson Prentice Hall, 2004. 
Dean, R. G. and Dalrymple, R. A.: Water wave mechanics for engineers and scientists, World Scientific, Singapore, 1991.

Dorfman, B. and Shemer, L.: Video image-based technique for measuring wave field evolution in a laboratory wave tank, Maritime Industry, Ocean Eng. and Coastal Resources, 2, edited by: Guedes Soares, C. and Kolev, P. K., Taylor \& Francis, London, 711-720, 2007.

Dysthe, K. B: Note on a modification to the nonlinear Schrödinger equation for application to deep water waves, Proc. R. Soc. Lond., A369, 105-114, 1979.

Hasimoto, H. and Ono, J.: Nonlinear modulation of gravity waves, J. Phys. Soc. Japan, 33, 805-811, 1972.

Hogan, S. J.: The potential form of the fourth-order evolution equation for deep-water gravity-capillary waves, Phys. Fl., 29, 34793480, 1986.

Kit, E. and Shemer, L.: Spatial versions of the Zakharov and Dysthe evolution equations for deep water gravity waves, J. Fluid Mech., 450, 201-205, 2002.

Lake, B. M., Yuen, H. C., Rungaldier, H., and Ferguson, W. E.: Nonlinear deep water waves: theory and experiment, 2. Evolution of a continuous wave train, J. Fluid Mech., 83, 49-74, 1977.

Lo, E. and Mei, C. C.: A numerical study of water-wave modulation based on a higher-order nonlinear Schrödinger equation, J. Fluid Mech., 150, 395-416, 1985.

Pelinovsky, E. and Kharif, C.: Simplified model of the freak wave formation from the random wave field, Proc. 15th Int. Workshop on Water Waves and Floating Bodies, edited by: Miloh, T. and Zilman, G. Caesaria, 142-145, 2000.
Shemer, L., Kit, E., Jiao, H.-Y., and Eitan, O.: Experiments on nonlinear wave groups in intermediate water depth, J. Waterway, Port, Coastal \& Ocean Eng., 124, 320-327, 1998.

Shemer, L., Jiao, H.-Y., Kit, E., and Agnon, Y.: Evolution of a nonlinear wave field along a tank: experiments and numerical simulations based on the spatial Zakharov equation, J. Fluid Mech., 427, 107-129, 2001.

Shemer, L., Kit, E., and Jiao, H.-Y.: An experimental and numerical study of the spatial evolution of unidirectional nonlinear waterwave groups, Phys. Fl., 14, 3380-3390, 2002.

Shemer, L., Goulitski, K., and Kit, E.: Evolution of wide-spectrum wave groups in a tank: an experimental and numerical study, Eur. J. Mech. B/Fluids, 26, 193-219, 2007.

Stiassnie, M.: Note on the modified nonlinear Schrödinger equation for deep water waves, Wave Motion, 6, 431-433, 1984.

Trulsen, K. and Dysthe, K. B.: A modified nonlinear Schrödinger equation for broader bandwidth gravity waves on deep water, Wave Motion, 24, 281-289, 1996.

Trulsen, K., Kliakhandler, I., Dysthe, K. B., and Velarde, M. G.: On weakly nonlinear modulation of waves on deep water, Phys. Fl., 12, 2432-2437, 2000.

Yuen, H. C. and Lake, B. M.: Nonlinear deep water waves: Theory and experiment, Phys. Fluids, 18, 956-960, 1975.

Zakharov, V. E.: Stability of periodic waves of finite amplitude on the surface of a deep fluid, J. Appl. Mech. Tech. Phys., 9, 190194, 1968. 